\title{
Ivan Sainsaulieu, Conflits et résistances au travail
}

Paris, Presses de Sciences Po, coll. « Contester », 2017

\section{Jean-Michel Denis}

\section{(2) OpenEdition}

\section{Journals}

Édition électronique

URL : https://journals.openedition.org/travailemploi/9931

DOI : 10.4000/travailemploi.9931

ISSN : 1775-416X

Éditeur

DARES - Ministère du Travail

Édition imprimée

Date de publication : 1 mars 2020

Pagination : 119-123

ISSN : 0224-4365

Référence électronique

Jean-Michel Denis, «Ivan Sainsaulieu, Conflits et résistances au travail », Travail et Emploi [En ligne], 161 | 2020, mis en ligne le 01 mars 2021, consulté le 27 septembre 2021. URL : http:// journals.openedition.org/travailemploi/9931 ; DOI : https://doi.org/10.4000/travailemploi.9931 


\title{
NoteS DE LECTURE
}

\section{Conflits et résistances au travail}

\author{
Ivan Sainsaulieu
}

Paris, Presses de Sciences Po, coll. «Contester », 2017, 184 p.

\section{Lu par Jean-Michel Denis*}

Le livre de Ivan Sainsaulieu Conflits et résistances au travail est le treizième ouvrage de la collection dirigée par Nonna Mayer consacrée aux multiples formes de la contestation politique et sociale. Il paraît près de dix ans après celui, inaugural, de Guy Groux et de Jean-Marie Pernot intitulé La Grève' ${ }^{1}$. Si l'objet de ces deux ouvrages n'est pas totalement identique, la grève n'étant qu'une des formes des conflits du travail, cette dernière publication permet néanmoins, indirectement, d'évaluer les mutations de la conflictualité au travail au cours de la période, et les transformations dans la manière de l'appréhender.

Ce livre part d'un constat: la persistance des conflits du travail qui atteste, selon l'auteur, de « l'échec de la pacification sociale » au sein de la sphère productive (p. 6) ${ }^{2}$, et d'un postulat: compte tenu de l'éclatement du salariat et de la différenciation des situations individuelles et collectives de travail, l'interprétation des conflits dans cette sphère à partir d'un cadre interprétatif unique serait devenu impossible. Plus nettement encore, il considère que l'analyse des conflits du travail souffre de l'utilisation de grilles de lecture qui déforment plus ou moins fortement la réalité. Il en repère trois qu'il range en deux catégories : la catégorie « objectiviste » rassemble les approches - sociologiques - qui soit survalorisent le conflit par la lutte des classes, soit le sous-estiment par la démocratie sociale; et celle, «subjectiviste», d'origine anglo-saxonne et davantage présente chez les politistes, dont l'approche trop micro, qu'elle soit compréhensive ou interactionniste, négligerait les configurations de travail.

Le livre d'I. Sainsaulieu trouve ici sa raison d'être. Si avec celui-ci, l'auteur obéit globalement aux consignes éditoriales de la collection qui est de proposer des ouvrages de synthèse sur un mode particulier d'action protestataire grâce à un travail de définition, de cadrage théorique et empirique, etc., il affirme surtout chercher à sortir l'analyse des conflits du travail de ces schémas préétablis. Comment? De deux manières. Premièrement, par une meilleure prise en compte des configurations

\footnotetext{
* Université Gustave Eiffel, Laboratoire Techniques, territoires et sociétés (Latts).

1. Groux G., Pernot J.-M. (2008), La Grève, Paris, Presses de Sciences Po.

2. L'année 2016, qui précède la parution de l'ouvrage, est de fait marquée par une hausse de la conflictualité symbolisée entre autres, au niveau interprofessionnel, par le mouvement contre la loi Travail - sources Dares.
} 
de travail qui entourent les conflits - souvent minorées dans l'analyse au profit de « logiques transversales, politiques ou multisectorielles » (p. 21) - via l'utilisation combinée de plusieurs modalités d'approches sociologiques (du travail, des organisations et des professions principalement); cette utilisation a pour conséquence de s'intéresser aux conflits au travail plutôt qu'aux conflits du travail. Deuxièmement, en privilégiant au conflit, que l'auteur considère comme un modèle figé, le concept de résistance, plus souple et mieux adapté pour caractériser les différentes formes d'oppression contemporaines; celles-ci étant multiples et ne relevant pas toutes d'une logique d'exploitation, il convient donc d'employer ce terme de résistance au pluriel. C'est à partir de cette proposition que sont structurés les cinq chapitres de l'ouvrage qui se présentent comme des « configurations de résistance ou de conflits au travail » (p. 22) différentes, quasi autonomes les unes par rapport aux autres, hormis les deux premières qui se succèdent logiquement.

En commençant le premier chapitre intitulé « Grèves et action syndicale » par le déclin des grèves, I. Sainsaulieu ne se contente pas de proposer une histoire à rebours du recours à la grève et de sa complémentarité avec le mouvement syndical, il décline cette histoire au passé. Sous-entendu : la grève comme marqueur essentiel de l'identité et de la révolte ouvrières appartient à un monde qui est en grande partie derrière nous; un monde dominé par le paradigme industriel dans lequel syndicalisme et action collective étaient indissociablement liés, au point même que le terme d'action collective s'est longtemps confondu avec celui de syndicalisme. L'élément intéressant de ce chapitre est justement de revenir sur la nature de ce lien, de le « dé-substancialiser ». Depuis leur origine en effet, le syndicalisme et la grève ont constitué deux corps, à la fois fusionnels et séparés, marquant de leur tension intrinsèque les multiples épisodes conflictuels de l'histoire du mouvement ouvrier. Signe supplémentaire de leur attachement, ces deux corps s'affaibliraient de concert, voire par effet réciproque : les syndicats banalisant la grève en l'utilisant comme vecteur de régulation sociale dans le jeu institutionnalisé des relations professionnelles; et la grève n'étant pas pour rien dans «la dynamique scissionniste au sein du syndicalisme français » (p. 48). La dépolitisation de la grève participerait également de l'affaiblissement de cette dernière, dépolitisation qui serait autant due à celle du monde du travail qu'à la séquence défensive dans laquelle sont embourbés les mouvements sociaux depuis plusieurs décennies. Toujours est-il que même si sa raréfaction - dans l'ensemble des pays industrialisés - n'est pas synonyme de disparition, la conflictualité au travail prendrait souvent aujourd'hui d'autres formes que la grève, comme veut le montrer l'auteur dans la suite de son ouvrage.

Dans le deuxième chapitre, la perspective se fait tourainienne. Pas seulement parce que I. Sainsaulieu se réfère à ce sociologue en qualifiant le conflit tertiaire de postindustriel mais aussi parce que ce basculement dans ce nouveau régime d'emploi et de travail le conduit à privilégier le concept de résistance au détriment de celui du conflit (de la même manière que la société programmée théorisée par Alain Touraine l'amenait à préférer le concept de mouvement social à celui de conflit relié à la société industrielle). Le développement des emplois tertiaires, qui placent en leur cœur la relation 
de service, leur polarisation et ses effets sur la précarisation des conditions d'emploi et de travail légitimeraient ce glissement en ce qu'ils reconfigurent très largement les « luttes et les résistances »- l'auteur revient justement sur les multiples combats menés ces dernières années par les femmes de ménage comme autant d'illustrations des « luttes précaires » (p. 63). Il en vient donc à s'interroger sur la particularité des conflits du tertiaire et à mettre l'accent sur le « retournement de sens de [du terme] service » comme arme des acteurs en lutte. Mais sans que l'on sache, pour rester sur ce questionnement autour de la spécificité de ces luttes, ce que fait précisément la relation de service au conflit. De même que l'on ne comprend pas le rapport que peuvent avoir ces conflits du tertiaire avec les mouvements d'occupation en France et à l'étranger sur lesquels l'auteur revient longuement dans ce chapitre.

Le chapitre trois consacré aux conflits des « salariés haut de gamme », c'est-à-dire les professionnels et les cadres, présente le même caractère décousu. Après avoir montré que l'insatisfaction au travail constitue le moteur principal des formes de résistance des salariés hautement qualifiés, I. Sainsaulieu passe sans crier gare à l'intensification du travail et aux effets qu'elle génère en termes de souffrance pour ces derniers. Une fois encore, ce sont moins les questions posées par l'auteur qui posent problème - le plus souvent pertinentes : pourquoi les problèmes de santé au travail n'entraînent que peu de conflits ? - que la structuration de sa présentation et son manque de précision. L'adoption de cette catégorie de «professionnels », dont on ne peut pas dire qu'elle soit très précise, le conduit à aborder des cas extrêmement disparates de salariés (les employés culturels, les professions libérales, les professionnels de la santé, les agents publics, etc.) « en résistance » contre des décisions et/ou des politiques elles-mêmes des plus diverses (problème d'emploi, de reconnaissance, refus des réformes budgétaires, opposition au nouveau management public, etc.) dont on ne perçoit pas réellement le point commun. En revanche, l'auteur énonce un point important sur cette question de la professionnalité: celui du recours insuffisant à la sociologie des professions dans l'approche combinée visant à analyser les mutations contemporaines du conflit et de ses acteurs. Alors qu'en la matière, le décloisonnement a surtout concerné la sociologie du travail (et des relations professionnelles) et celle des mobilisations, il conviendrait effectivement de mobiliser davantage les apports de cette dernière.

S'intéresser aux conflits et aux différentes formes de résistance dans le domaine du travail requiert également de s'intéresser à ceux qui ne s'inscrivent pas dans le rapport verticalisé de la subordination. Mais le fait que ces « discordes » (conflits entre personnes dans le cadre du travail pour des motifs divers : opinions, intérêts, culturels, etc.) puissent prendre également pour cadre le travail les transforme-t-elles en conflits du même nom ? C'est ce que pense en tout cas l'auteur qui leur consacre l'avant-dernier chapitre de son livre. En sociologue et non en psychologue, en plaçant l'origine du conflit non pas dans l'homme mais dans l'organisation. Après avoir défendu l'apport de la sociologie des professions à l'étude des conflits du travail, il plaide ici pour celui de la sociologie des organisations, grâce auquel il devient possible de dresser des typologies des conflits en les reliant aux différents contextes organisationnels 
dans lesquels ils s'inscrivent. Lui-même fait le lien, à travers cette proposition, avec le modèle des «mondes sociaux de l'entreprise » de Renaud Sainsaulieu ${ }^{3}$.

La dernière thématique abordée par I. Sainsaulieu, sans qu'il s'agisse réellement d'une «configuration de résistance ou de conflit au travail» boucle avec le début de l'ouvrage consacré au déclin de la grève et du syndicalisme. Là encore, elle est introduite par une interrogation: "Qu'advient-il de la politisation au travail sans ses moteurs que sont le militantisme et le conflit? » (p. 129). Cette expression de « politisation au travail » n'est pas des plus claires, qu'il s' agisse des termes choisis ou de leur association. Que désigne précisément la politisation et qu'est-ce qui est censé relever du ou de la politique « au travail »? On comprend rapidement malgré tout que, par politisation, il faut entendre conscience de classe et le fait que, pendant longtemps, « les représentations politiques ont été [...] marquées par l'appartenance à des positions sociales » (p. 131) dans l'espace du travail; d'où le caractère politique des conflits du travail. Qu'en est-il aujourd'hui alors que la conscience de classe ne présente plus de caractère unifié, au point d'avoir été réduite à sa plus simple expression (conscience individuelle et morale $)^{4}$ ? Les résistances au travail expriment-elles encore des valeurs collectives et ces valeurs s'ancrent-elles encore dans le travail ? S'il est difficile de répondre à ces questions, c'est que ces valeurs ne semblent plus (autant?) s'incarner dans les institutions ${ }^{5}$, ce qui ne signifie pas nécessairement qu'elles aient disparu. D'où la recommandation de l'auteur d'adopter une démarche quasi anthropologique afin de faire ressortir, derrière les multiples formes de révoltes et de résistance, les « rapports ordinaires » noués par les salariés au politique.

En définitive, malgré son caractère désordonné, ce livre est intéressant pour les questions qu'il pose et pour son appel à une plus grande synergie des approches sociologiques dans l'étude des conflits et des formes de résistance contemporaines. Curieusement, il n'y intègre pas l'apport de la sociologie des relations professionnelles à qui il reproche sa vision fonctionnelle et institutionnelle du conflit qui n'aurait d'autre visée que de servir le « compromis négocié dans le cadre du dialogue social » (p. 12). Il s'agit là d'une singulière réduction des orientations théoriques et programmatiques de ce sous-champ, qui ne prend pas en compte les multiples recherches conduites ces dernières années sur les mutations de la conflictualité au travail, ne serait-ce que les travaux menés dans le cadre du Réseau thématique 18 (Relations professionnelles) de l'Association française de sociologie. Par ailleurs, la recommandation de I. Sainsaulieu de faire davantage attention à la contextualisation de la conflictualité au travail, pour

3. Osty F., Sainsaulieu R., Uhalde M. (2007), Les Mondes sociaux de l'entreprise. Penser le développement des organisations, Paris, La Découverte.

4. Comme le pense François Dubet (p. 47): «Dans le régime des inégalités multiples, l'expérience et la critique des inégalités se détachent des catégories collectives qui les enchâssaient et sont d'abord vécues comme des expériences personnelles, singulières et intimes. Pour en témoigner, chacun parle d'abord de soi et de sa souffrance », «La transformation des colères en politiques est-elle possible?», in AOC [Analyse Opinion Critique] (2019), «"Gilets jaunes." Hypothèses sur un mouvement », AOC Cahier \#1, Paris, La Découverte.

5. Y compris de leur propre fait. À ce sujet, voir Quijoux M., Yon K. (2018), « Syndicalisme et politique: dé-liaisons dangereuses? », Savoir/Agir, n 45, pp.7-11. 
importante qu'elle soit, n'est pas foncièrement nouvelle. Un dispositif d'enquête comme celui proposé par la Direction de l'animation de la recherche, des études et des statistiques (Dares) avec REPONSE (Relations professionnelles et négociations d'entreprise), qui existe depuis le début des années 1990, offre par exemple les possibilités de cette contextualisation du conflit (économique, organisationnelle et sociale), d'autant qu'il intègre des post-enquêtes par voie monographique qui permettent d'aller au plus près du lieu dans lequel il se développe. C'est entre autres cette enquête qui a montré, grâce à des données empiriques à large échelle, que le conflit au travail ne se réduisait pas à la grève et qui permet aujourd'hui à I. Sainsaulieu de reprendre, après d'autres, le concept de résistance au travail.

Doit-on désormais le préférer à celui de conflit? À la fois pour échapper à la vision moniste souvent générée par son utilisation et pour privilégier l'étude au plus près des conduites sociales au travail? Sans nécessairement rappeler que toute conduite informelle n'est pas résistante et que toute résistance ne remet pas nécessairement en cause l'ordre productif en vigueur (rappel qui vaut d'ailleurs également pour le conflit) ${ }^{6}$, on peut y voir deux risques : le premier, que l'on retrouve dans l'ouvrage de I. Sainsaulieu, est de produire un catalogue large et indifférencié de résistances au travail très diverses (quant à leurs formes, motivations, acteurs, etc.), rendant de ce fait très difficile toute tentative de taxinomie et de conceptualisation alors que c'est justement cet exercice qui permet de déceler les logiques sociales et politiques communes qui les conditionnent et d'en dégager le sens. Le second est relatif à la représentation politique que l'on continue à avoir du terme de résistance, et qui contamine dans une certaine mesure l'usage que l'on peut en avoir dans le champ du travail. Qu'on le veuille ou non, est assimilée à ce terme l'image d'un combat le plus souvent éparpillé, clandestin et surtout essentiellement défensif. Or, que la conjoncture actuelle des conflits au travail soit, sans discussion, de cette nature ne signifie pas qu'elle le demeure à jamais.

6. Bouquin S. (2011), «La question des résistances au travail dans la sociologie du travail française », Actuel Marx, $n^{\circ} 49$, pp. 60-72. 